\title{
Hand-Shaped Force Interface for Human-Cooperative Mobile Robot
}

\author{
Riku Hikiji \\ Shuji Hashimoto \\ Department of Applied Physics, \\ School of Science and Engineering, \\ Waseda University \\ 3-4-1 Okubo, \\ Shinjuku-ku, Tokyo 169-8555 JAPAN \\ +81352863233 \\ \{riku/shuji\}@shalab.phys.waseda.ac.jp
}

\begin{abstract}
Aiming at realization of direct and intuitive cooperation between human and robot, we propose an interface system for a two-wheeled, autonomous mobile robot that can take physical communication with its user via haptic, or hand-tohand force interaction. The hand-shaped device equipped on the robot has $1 \mathrm{DOF}$ at the finger part and is capable of gently grasping a human hand when actuated. The force information is acquired by using strain gages on the flexible, rubber-made arm that physically supports the hand. The robot's motion is determined by the force input and/or the environmental condition. The robot recognizes obstacles by using bumper sensors and ultrasonic sensors around the body. When it touches an obstacle, the robot changes the route regardless of the user's intentional force and thus informs the user of such situation of obstacle avoidance. In this research, we design simple algorithms for both human-following and human-leading motions, and devise experiments with human users. Qualitative and quantitative evaluations of the experimental results are also presented.
\end{abstract}

\section{Keywords}

Human-machine interface, human-cooperative robot, haptic interface, "Kansei", human-following, human-leading, force interaction

\section{INTRODUCTION}

Until recent days, tasks of robots had been mostly limited to heavy labors and repetitive works in industrial factories, extreme circumstances and so on. From now on, however, robots are required to hold more and more works in fields of nursing, aiding, communication, entertainment, etc. Their operational opportunities in human environment are increasing significantly, and the stance of robots is shifting from "in place of human" to "with human".

An operation in human environment, in most cases, requires massive transactions of dynamic environmental recognition, sound recognition, linguistic recognition and motion planning. There are many technological problems to be overcome for self-controlled autonomous mobile robots that work without acquiring any support from others. It seems to require more time until the appearance of such robots. On the other hand, robots that can work as acquiring support from human are considered to be more practical at present. Studies on human-cooperative robots are drawing considerable attention of robotics researchers.

In human-robot cooperation, it is important to develop an interface system that affords direct and intuitive interactive communication. There are papers on transferring task by cooperation of multiple mobile robots [1] or on cooperative carrying task by a human and a manipulator robot [2,3]. Human-following experiment with biped humanoid robot is reported as well [4]. In our laboratory, some studies have been proposed to realize haptic interaction between users in distance $[5,6]$. All of the above utilize force information in achieving cooperative tasks or communication, but very few of them are essentially designed to be human interface. Efficient interface system for human-robot cooperation must afford, or appeal to "Kansei" of, human users to interact with the robot. "Kansei" is a human ability of achieving perception in non-logical way [7]. The study of interface system utilizing force [8] suggests that handling, safety and impression are also important factors. By the way, one of our most natural and well-regarded communication ways is to take hands of each other. Handto-hand interaction provides direct physical force information as well as an effect on mental side in terms of togetherness, amiability, and security. It seems efficient to 
utilize haptic interaction in an interface system of humancooperative robot.

Thus we propose the hand-shaped force interface for the autonomous mobile robot that is designed to take physical communication with the user via haptic/force interaction. The hand-shaped device has 1 DOF at the finger parts and is capable of gently grasping a human hand when it is actuated. The force information is acquired by the strain gages that are attached on the flexible rubber-made arm physically supporting the hand. The robot's motion is determined by the force input and/or the environmental condition. Fundamental obstacle recognition is achieved by using bumper sensors and ultrasonic wave sensors around the body. The robot informs the user of obstacles he/she is not aware of by changing the route regardless of the intentional force. Simple algorithms for both humanfollowing and human-leading tasks are designed. We devise experiments with human users, presenting qualitative and quantitative evaluation to examine the system's efficiency.

In the future, the system can be applied to a guide robot to be used in various scenes, a communication robot for children and elders, and a performance robot in achieving interaction with a human such as dance.

\section{SYSTEM}

This section explains the structure and function of the interface system in application to the human-cooperative mobile robot. First, we introduce the base mobile robot. Then, we view the whole system and function of the proposed force interface.

\section{Robot Body}

The robot we use in this research is two-wheeled mobile robot that can move forward/backward and rotate clockwise/counter-clockwise. Equipped obstacle sensors are bumper sensors and ultrasonic wave sensors. The bumper sensors are equipped in front and on the tail of the body and can sense obstacle contact in six different directions. The ultrasonic sensors are mounted to detect obstacles in front of the robot without touching.

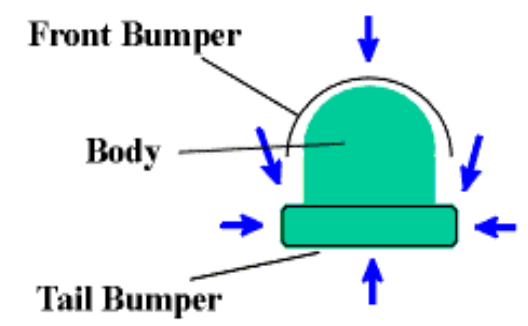

Fig.1 Equipment of the bumper sensors and directions of obstacle sensing (Top View)

\section{Interface Structure}

Appearance of the whole robot is shown in Fig.2. The haptic, or force, interface system is composed of the hand- shaped device supported by the flexible arm. The hand part is made of a plastic skeleton covered with a rubber glove, and is capable of gentle grasp with $1 \mathrm{DOF}$ at the fingers. When the hand part is grasped, it is actuated to grasp back the human hand. The arm part is made of two rubber sticks, one vertically fixed on the top of the robot body and the other horizontally on top of the vertical one.

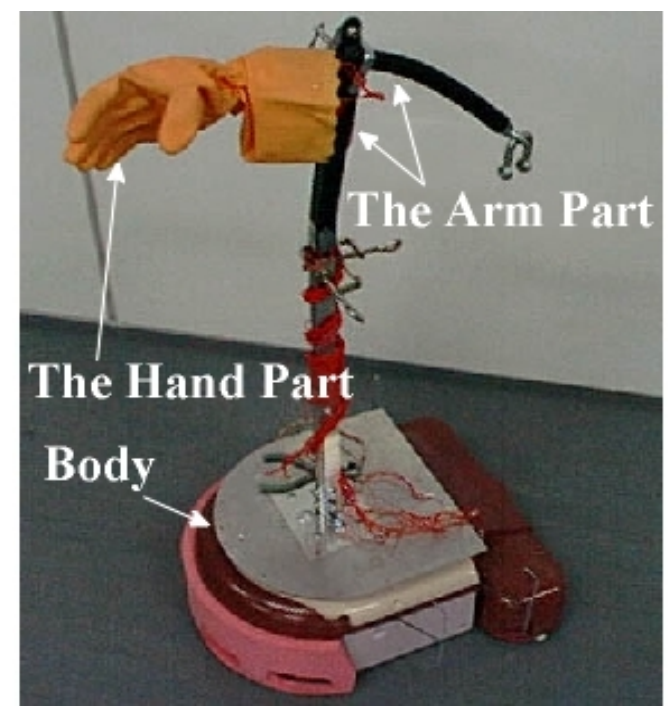

Fig.2 Outlook of the robot with the Hand-Shaped Force Interface

\section{The Arm Part and Force Sensing}

The arm part physically supports the hand part, and since it is rubber-made, it can easily be bent as an intentional force is exerted to the hand part. Flexibility of the arm thus provides a structural compliance to the system.

With the use of bridged strain gages, the arm part also functions as a force sensor. We adopt the Four Active Gage Method for measuring the force/torque. Each set of the two bridges (one on the vertical part of the arm and the other on the horizontal part) provides an independent output corresponding to the bend in a particular direction, that is, either forward/backward or clockwise/counter-clockwise (Fig.3). Independence as well as linearity of the force sensor output is confirmed in experiment as shown in Fig.4.

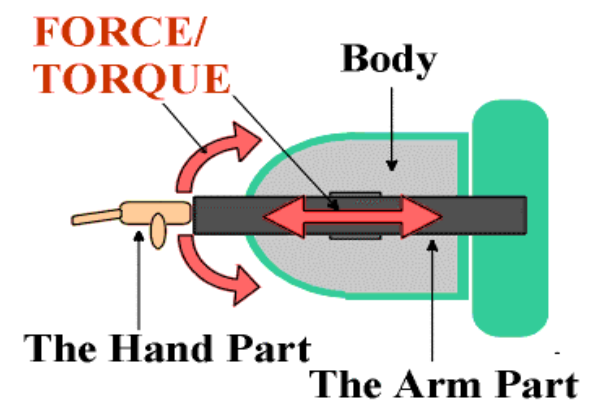

Fig.3 Decomposed intentional force exerted to the arm part (Top View) 


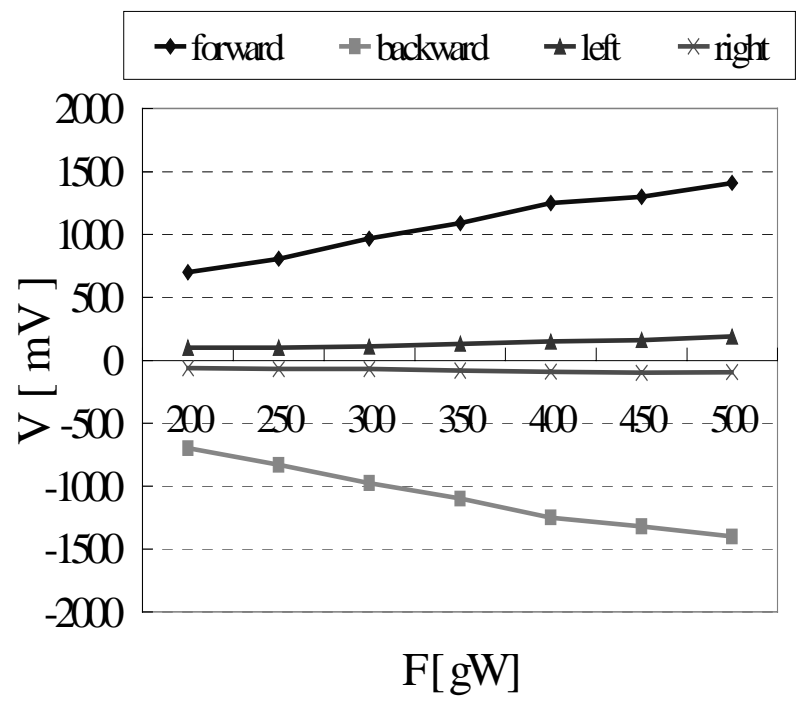

Fig.4 Bend sensor output when force is exercised either forward, backward, to the left, or to the right

\section{The Hand Part and Grasping}

On the bottom side of the hand is a micro-switch as a human grasp sensor (Fig.5). When the hand part is grasped, the micro-switch is turned on, and the fingers are actuated to gently grasp back the human hand. We implemented an electro-thermal actuator (BMF250, Toki Corporation [9]). It is made of threadlike Shaped Memory Alloy (SMA). It contracts like muscles when electric current flows, and it elongates when cooled. The 1 DOF fingers are directly attached to the actuator as shown in Fig.6.

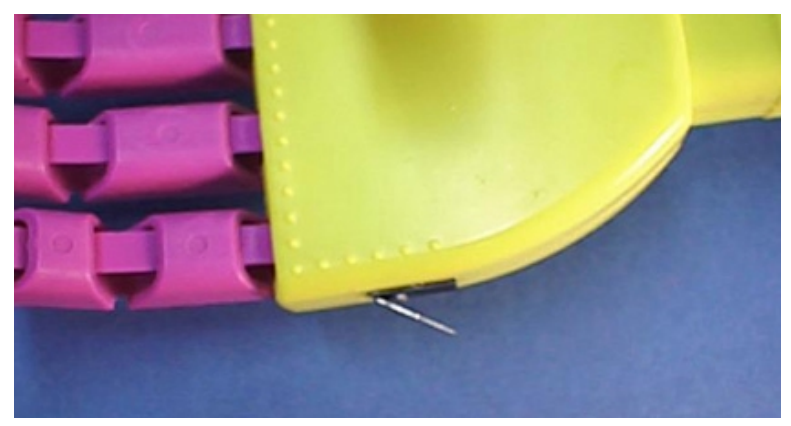

Fig.5 The micro-switch sensor on the bottom side of the Hand Part

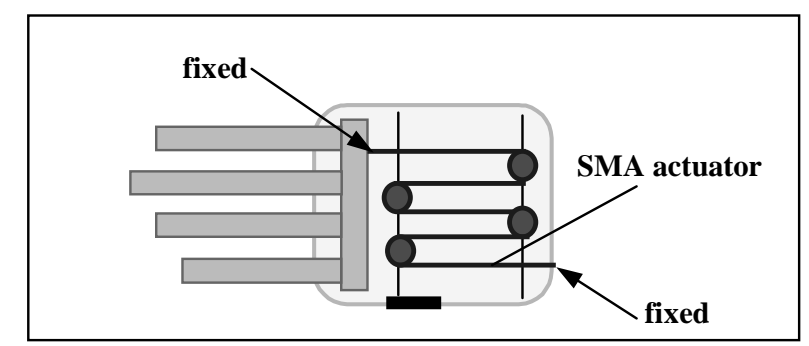

Fig.6 The Structure of SMA actuator

\section{CONTROL}

This section describes how to control the whole robotic system with the proposed interface.

\section{Control Structure}

The intentional force exerted to the interface system gives the set point of the robot's mobilization control. Fig.7 shows the entire structure of the motion control system. The algorithm is described in the following section.

Control of the grasp mechanism is open-looped, and strength of the grasping is determined experimentally.

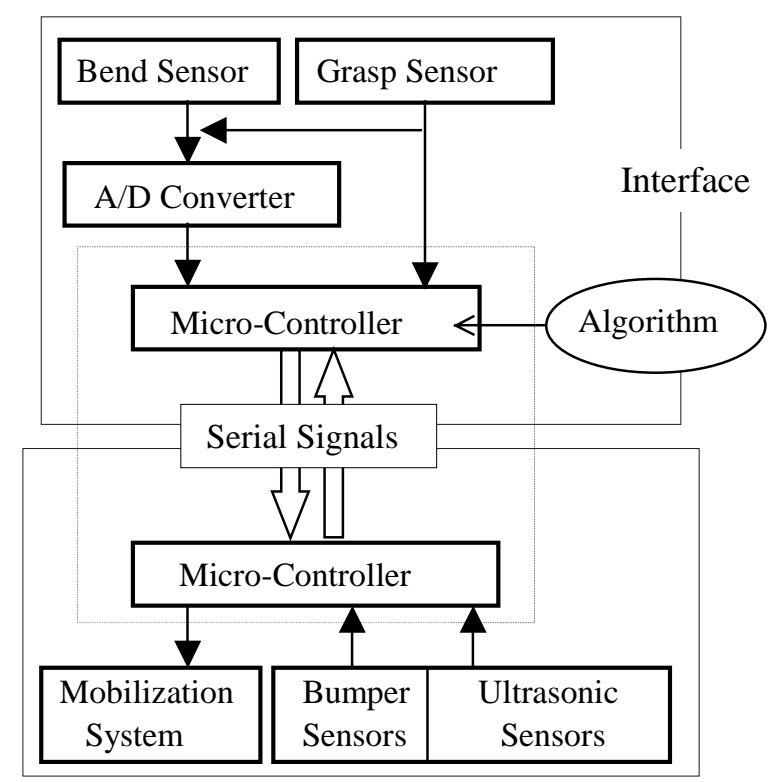

Fig.7 Diagram of the control structure

\section{Algorithm}

We have developed two different algorithms, one for human-following task and the other for human-leading task. With the human-following algorithm, the robot moves so as to cancel out the intentional force exerted to the handshaped interface (Fig.8). With the human-leading algorithm, route of the robot's leading task is pre-programmed, and the robot executes the task unless excessive counter-directional force is exerted (Fig.9). When the human follower pulls the robot's hand toward opposite direction of the leading motion, the robot stops until the intentional force ceases, meaning the follower can catch up delay. In both algorithms, when the robot touches obstacle, it executes "obstacle avoidance motion (Fig.10)" regardless of the intentional force input by the human partner. Since the robot and the partner are taking hands of each other, force information can be directly communicated, and thus the robot can provide the obstacle information to the partner. The robot and the human can avoid obstacles cooperatively even in case of the human not aware of obstacles. 
In complex environment, it is possible that robot finds obstacle in different direction at the same time. When two or more conflicting "obstacle avoidance motion" occurs, for example when both right and left bumper sensors find obstacle, the robot will stay still for a second and wait for human assistance so that it can avoid vibratory motion.

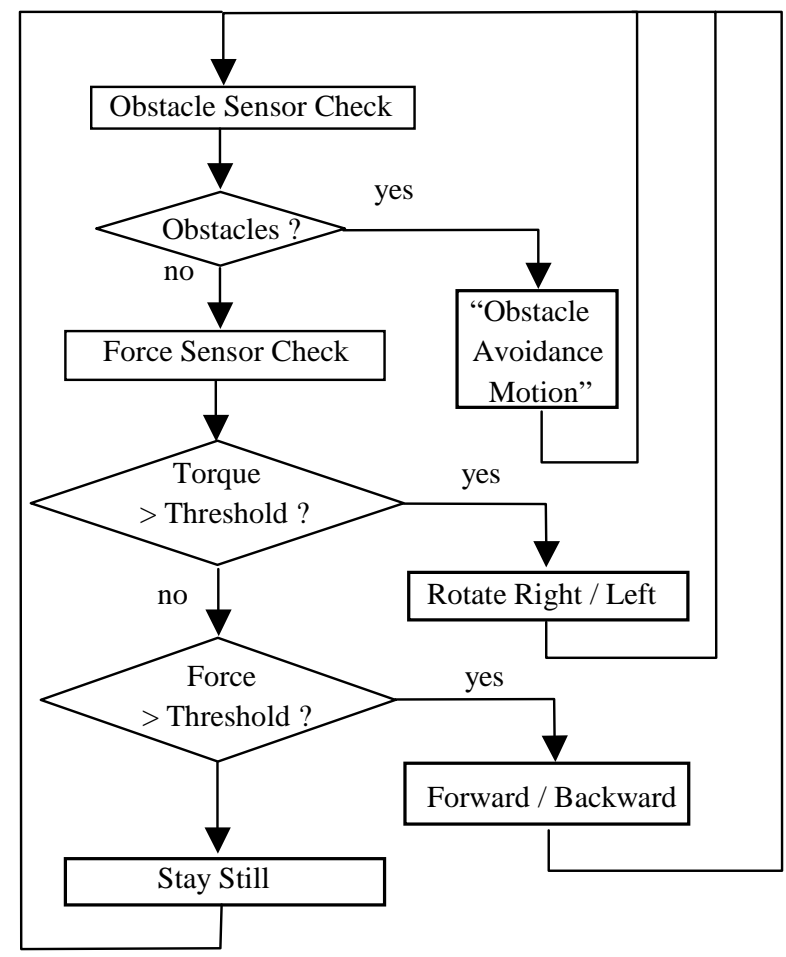

Fig.8 Algorithm flow chart of human-following task

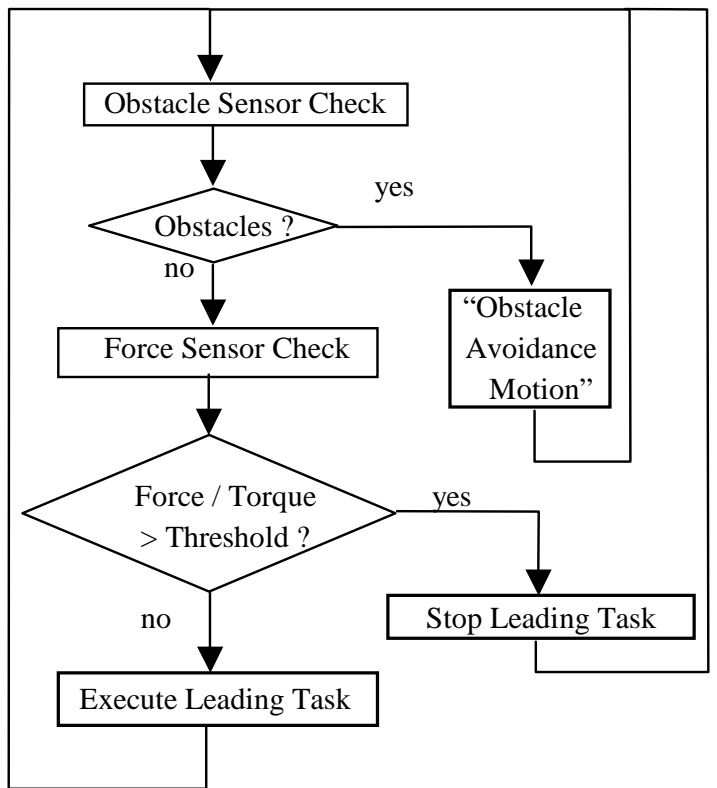

Fig.9 Algorithm flow chart of human-leading task

\begin{tabular}{|l|l|}
\hline $\begin{array}{l}\text { Direction of } \\
\text { force/torque }\end{array}$ & $\begin{array}{c}\text { Respective motion } \\
\text { of the Robot }\end{array}$ \\
\hline $\begin{array}{l}\text { front } \\
\text { left-front } \\
\text { right-front }\end{array}$ & move backward for about $1[\mathrm{sec}]$ \\
\hline left & rotate counter-clockwise for $30[\mathrm{deg}]$ \\
\hline right & rotate clockwise for $30[\mathrm{deg}]$ \\
\hline tail & move forward for about $1[\mathrm{sec}]$ \\
\hline
\end{tabular}

Fig.10 "Obstacle avoidance motion"

\section{EXPERIMENT}

In order to examine the efficiency of the proposed interface, 3 different experiments are devised.

\section{Human-Following Experiment}

In this experiment, the human user leads the robot from the start point to the goal point in two-dimensional static environment. A motion capture system is used to acquire the trajectories of the human and the robot (Fig.11) and the fluctuation of the distance between them during the task (Fig.12). These results support the achievement of elementary human-following task.

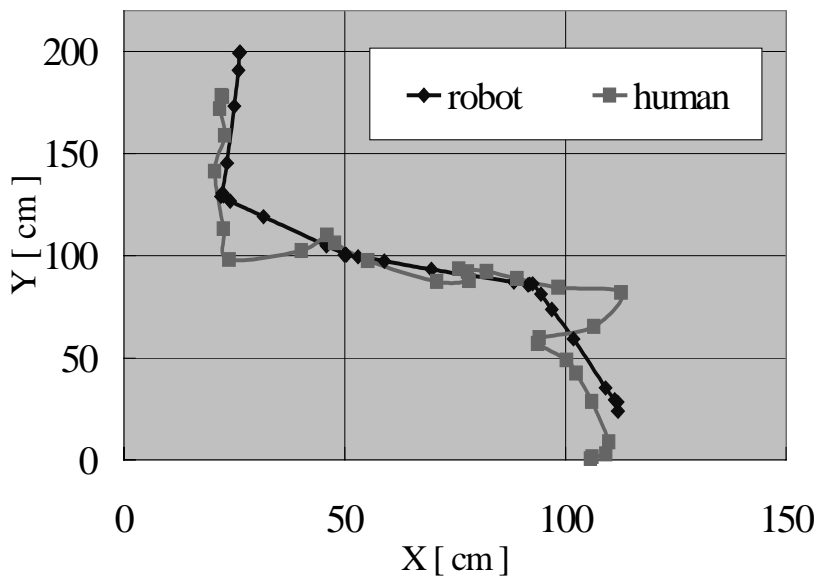

Fig.11 Trajectories of the human and the robot in the HumanFollowing Experiment

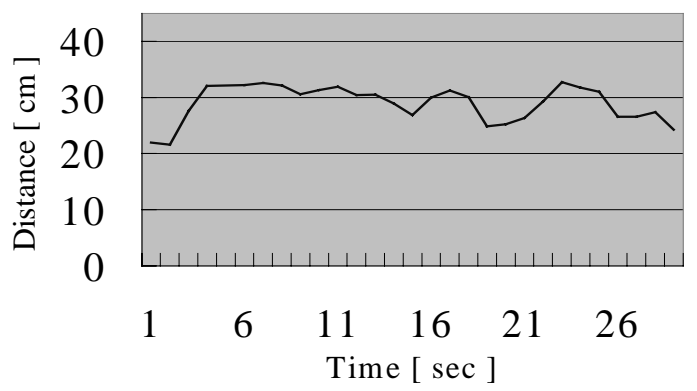

Fig.12 The distance between the human and the robot in the Human-Following Experiment 


\section{Human-Leading Experiment}

In this experiment, the human volunteers are requested to follow the robot's lead with an eye mask on. The robot is programmed to execute the human-leading task in the experimental environment as shown in Fig.13. The average goal time of the human-leading tasks of 10 volunteers is comparable to the goal time of the robot moving by itself without human follower (Fig.14). This suggests that an effective human-leading task is achieved. Result of the questionnaire after the experiment supports our proposition as well (Fig.15)

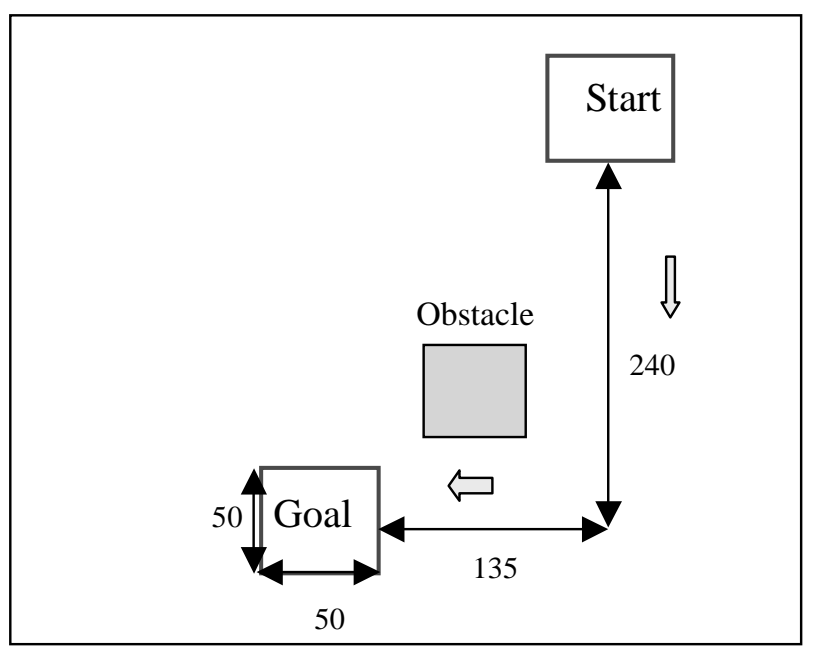

Fig.13 Environmental map of the Human-Leading Experiment

\begin{tabular}{|l|c|}
\hline $\begin{array}{l}\text { Average goal time of } 10 \text { volunteers } \\
\text { when led by the robot }\end{array}$ & 29 [sec] \\
\hline $\begin{array}{l}\text { Goal time of the robot when moving } \\
\text { by itself without human follower }\end{array}$ & 23 [sec] \\
\hline
\end{tabular}

Fig.14 Goal time of the tasks with and without human follower

\begin{tabular}{|l|l|c|}
\hline & Yes & No \\
\hline $\begin{array}{l}\text { 1) was able to feel intentional force } \\
\text { from the leading robot }\end{array}$ & 10 & 0 \\
\hline 2) felt securely lead to the goal & 8 & 2 \\
\hline
\end{tabular}

Fig.15 Questionnaire answers of the Human-Leading Experiment

\section{Comparative Experiment of Interface}

In order to evaluate the efficiency of the interface system, a comparative experiment is also held with the help of the same 10 volunteers. Two other different types of the existing interface devices along with the hand-shaped force interface are used in comparison. In the same experimental environment as shown in Fig.13, this time, the volunteer users are requested to lead the robot from the start to the goal. Two of the existing interface devices are a digital joystick and a remote controller. Each interface device, including the hand-shaped force interface, is handed to the user without any instruction. Leading tasks begin when the user confidently feels that he/she has learned enough to handle the robot with each interface. The average goal time of all users suggests that the hand-shaped force interface is useful in executing such task (Fig.16). Afterwards, questionnaire on qualitative evaluation is held. In each category, users must rank the interfaces in order of quality. Scores are given in integers from 3 (best) to 1 (worst), and none of the scores must be repeated more than once. The result supports that the newly designed interface excels in all factors of human interface, especially in affordance, or "Kansei" appeal, and impression. Handling seems also as efficient as other two (Fig.17).

\begin{tabular}{|c|c|c|c|}
\hline \multicolumn{4}{|c|}{$\begin{array}{l}\text { (a) The hand-shaped force interface } \\
\text { (b) joystick } \\
\text { (c) remote controller }\end{array}$} \\
\hline Type of Interface & (a) & (b) & (c) \\
\hline Average goal time & 39 & 30 & 45 \\
\hline
\end{tabular}

Fig.16 Average goal time of using different interfaces in comparative experiment

\begin{tabular}{|c|ccc|}
\hline CATEGORY & (a) & (b) & (c) \\
\hline $\begin{array}{c}\text { Was able to handle } \\
\text { (1) intuitively } \\
\text { ("Kansei" appeal) }\end{array}$ & 2.6 & 1.9 & 1.5 \\
\hline $\begin{array}{c}\text { Had good handling } \\
\text { (2) of the whole robot } \\
\text { (Handling) }\end{array}$ & 2.1 & 2 & 1.9 \\
\hline $\begin{array}{c}\text { Felt affinity } \\
\text { (3) or amiability } \\
\text { (Impression) }\end{array}$ & 2.9 & 2 & 1.1 \\
\hline
\end{tabular}

Fig.17 Average scores of the questionnaire comparative experiment

\section{COCLUSION}

In this paper, the hand-shaped force interface for humancooperative mobile robot is proposed. By utilizing hand-tohand force interaction, profuse communication with intentional force between a human and a robot is achieved. In the human-following task, the robot not only follows the human user to the direction in which the intentional force is exerted, but also recognizes obstacles and communicates that information to the user. In the human-leading task, the robot moves as it is pre-programmed. It stops when the 
human follower exerts intentional force to the opposite direction of its motion. As for evaluation of the proposed robotic system, we experimented on both tasks in real human-robot cooperation. Efficiency of the system as a human interface is also testified in comparison to other interface systems. The experimental results suggest that the proposed system fulfill the important requirements of human interface.

Now, we are planning to apply a velocity/acceleration control to the robot for achieving more smooth motion. We are also considering on supplementing utilization of sound information for more informative communication between a human and a robot in order to develop multi-modal robot interface.

\section{REFERENCES}

1. J. Ota, Y. Buei, T. Arai, H. Osumi and K. Suyama, "Transferring Control by Cooperation of Two Mobile Robots", Journal of the Robotics Society of Japan, Vol.14 No.2, 263-270, 1996 (in Japanese).

2. K. Kosuge and N. Kazamura, "Control of a Robot Handling an Object in Cooperation", Proc. of IEEE International Workshop on Robot and Human Communication, 142-147, 1997.

3. R. Ikeura and H. Inooka, "Variable Impedance Control of a Robot for Cooperation with a Human", Proc. of
1995 IEEE International Conference on Robotics and Automation, 3097-3102, 1995.

4. J. Yamaguchi, S. Gen, S. A. Setia Wan and A. Takanishi, "Interaction between Human and Humanoid through the Hand Contact", Proc. of 16th Conference of the Robotics Society of Japan, 951-952, 1998 (in Japanese).

5. K. Ouchi and S. Hashimoto, "Handshake Telephone System to Communicate with Voice and Force", Proc. of IEEE International Workshop on Robot and Human Communication, 466-471, 1997.

6. Y. Fujita and S. Hashimoto, "Experiments of Haptic and Tactile Display for Human Telecommunication", Proc. of the 8th IEEE International Workshop on Robot and Human Interaction (RO-MAN'99), 334-337, 1999.

7. S. Hashimoto, "KANSEI as the third target of information processing and related topics in Japan", KANSEI The Technology of Emotion AIMI International Workshop proceedings, 101-104, 1997

8. J. Yokono and S. Hashimoto, "Center of Gravity Sensing for Motion Interface", Proc. of IEEE International Conference on Systems, Man and Cybernetics, 1113-1118, 1998.

9. Toki Corporation Official Website. Available at http://www.toki.co.jp/BioMetal/_index.html. 